\title{
A mathematical model for atherosclerotic plaque formation and arterial wall remodelling
}

\author{
Md Hamidul Islam ${ }^{1} \quad$ P. R. Johnston ${ }^{2}$
}

(Received 22 December 2015; revised 25 October 2016)

\begin{abstract}
Atherosclerosis is a condition whereby fatty material is deposited in the walls of arteries (plaque) resulting in a thickening of the wall. Here we develop a mathematical model describing the biochemical processes of the formation of atherosclerotic plaque, which involves the interaction between pro-inflammatory mediators, modified low density lipoprotein, monocytes, macrophages, foam cells and high density lipoprotein. In addition, based on the outcomes of the biochemical model, we develop a plaque growth model that takes into account both the inward and outward expansion of the arterial walls. We examine the stability and bifurcations of this model in order to explore the clinical and medical implications of plaque growth.
\end{abstract}

DOI:10.21914/anziamj.v57i0.10386, (c) Austral. Mathematical Soc. 2016. Published November 10, 2016, as part of the Proceedings of the 12th Biennial Engineering Mathematics and Applications Conference. ISSN 1445-8810. (Print two pages per sheet of paper.) Copies of this article must not be made otherwise available on the internet; instead link directly to the DOI for this article. Record comments on this article via http://journal. austms.org.au/ojs/index.php/ANZIAMJ/comment/add/10386/0 


\section{Contents}

1 Introduction

C321

2 The mathematical model

C323

2.1 Biochemical model . . . . . . . . . . . . . . . . . C323

2.2 Plaque Growth Model . . . . . . . . . . . . . . . C C330

3 Results and discussion

C333

3.1 Simulations of the dynamics . . . . . . . . . . . C C333

3.2 Bifurcation analysis . . . . . . . . . . . . . . C337

3.3 Plaque growth simulations . . . . . . . . . . . . . C C339

4 Conclusions

C340

References

C342

\section{Introduction}

Atherosclerosis is a chronic inflammatory disease, which deposits fatty material inside the walls of arteries (plaque), resulting in a thickening of the intimal region. These lesions can form in the intima, the inner most layer of the arterial wall, as early as infancy and remain subclinical for decades without causing any clinical symptoms. The lesions may regress over time or can continue to grow and cause sudden clinical manifestations such as coronary artery disease, ischemic cerebrovascular disease, and peripheral heart disease. The artery wall initially dilates in response to the thickening so that the blood flow through the lumen remains unaltered [1]. However, at some point, the artery can no longer compensate by dilation and the plaque starts to grow towards the lumen, which may eventually cause stenosis or occlusions. Besides the hemodynamic effects on blood flow and blood pressure, these stenosed plaques often weaken the arterial wall, leading to rupture and the 
sudden occlusions of blood flow to vital organs. Plaques with a high lipid content, that is, a large necrotic core in their centre and with a thin fibrous cap, are more susceptible to rupture [2].

A dysfunctional endothelium, which is characterised as an endothelium with increased endothelial cell death or injury and impaired repair capacity, plays a pivotal role in initiating atherosclerosis. Experimental studies suggest that the endothelial systems in patients with hypercholesterolaemia and hypertension may be exposed to repetitive injury, and have reduced repairing capacity compared to normolipidemic patients with healthy normotensive controls [3, 4]. Leukocytes (white blood cells such as monocytes and lymphocytes) are recruited primarily to the site of injury to serve as reparative inflammatory cells [5]. However, due to repeated injury and a prolonged repair process, low density lipoprotein (LDL) may invade the intima where they rapidly become oxidised. In the presence of modified LDL (ox-LDL) inside the intima, the primary reparative inflammatory monocyte cells now serve as inflammatory mediators and these are fundamental to the development of an atherosclerotic lesion.

Monocytes first differentiate themselves into macrophages and then consume ox-LDL. Being stimulated by the consumption of ox-LDL, macrophages initiate a series of intracellular events, such as secretion of monocyte chemotactic protein 1 (MCP-1), pro-inflammatory cytokines IL-1 and endothelium stimulating cytokines, such as tumor necrosis factor $\alpha(\mathrm{TNF} \alpha)[7,6]$. These chemoattractants promote further immigration of monocytes, which may eventually lead to a chronic inflammatory process. Macrophages that have consumed ox-LDL, become foam cells and collectively form fatty streaks inside the intima. On the other hand, high density lipoprotein (HDL) inhibits the progression of atherosclerosis by removing cholesterol from foam cells, as well as by limiting the inflammatory processes that promote atherosclerosis [8].

Atherosclerotic plaques initiate and grow through a number of nonlinear processes operating on different timescales and in different parts of the tissue. Some of these processes occur in the endothelial layer whereas others occur in 
the arterial wall. Therefore, mathematical and computational models are a natural framework to provide an integrated description of the disease, which can help develop therapeutic hypotheses. The research in this area to date is limited and wide open to new ideas. There are several mathematical studies which looked at atherosclerosis as an inflammatory disease [9, 10, 11], with most only considering the interactions between monocytes, macrophages, ox-LDL and foam cells. More recently, several mathematical models included the interactions between the above-mentioned cells as well as HDL and a number of other cellular species $[12,13]$. However, none of these studies considered both the inward and outward expansion of the artery walls in their plaque growth models.

We consider atherosclerosis as a continuous process and propose a biochemical model containing a system of ODEs to simulate the dynamics of the formation of an atherosclerotic plaque. We examine the stability and bifurcations of this model in order to investigate the conditions for an inflammatory lesion to be initiated and under what circumstances it may progress or regress over time. Another novel aspect of this study is the inclusion of the inward and outward expansion of the arterial walls in a plaque growth model.

\section{The mathematical model}

\section{$2.1 \quad$ Biochemical model}

To model the early stages of atherosclerosis we use the description of the mechanisms outlined in Section 1. We model the time evolution of the mass concentrations of six species: inflammatory mediators; monocytes; macrophages; ox-LDL; foam cells; and HDL. Hence, the model comprises equations for:

$c(t)$ concentration of pro-inflammatory mediators $\left(\mathrm{pg} \mathrm{mm}^{-3}\right)$; 
$m(t)$ concentration of monocytes $\left(\mathrm{pg} \mathrm{mm}^{-3}\right)$;

$\mathrm{M}(\mathrm{t})$ concentration of macrophages $\left(\mathrm{pg} \mathrm{mm}^{-3}\right)$;

$\mathrm{L}_{\mathrm{ox}}(\mathrm{t})$ concentration of oxidised LDL $\left(\mathrm{pg} \mathrm{mm}^{-3}\right)$;

$\mathrm{F}(\mathrm{t})$ concentration of foam cells $\left(\mathrm{pg} \mathrm{mm}^{-3}\right)$; and

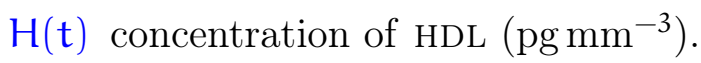

None of these six species are present before endothelial injury, hence the initial conditions are

$c(0)=0, \quad m(0)=0, \quad M(0)=0, \quad L_{o x}(0)=0, \quad F(0)=0, \quad H(0)=0$.

Atherosclerosis risk factors, such as hypertension, hypercholesterolaemia, diabetes, vascular aging and estrogen deficiency, are known to impact the health of the endothelium and its integrity [3]. Accordingly, the endothelial system in patients with one or more of these risk factors experiences abnormal endothelial cell turnover (or injury) and delayed replication [4]. Damage to the endothelium leads to the production of a number of inflammatory mediators, such as cell adhesion molecules, cytokines, chemokines and growth factors. These inflammatory mediators direct the recruitment of inflammatory cells at the site of the injury as a reparative mechanism. However, these same inflammatory mediators also play a fundamental role in the development of atherosclerotic lesions.

To model the concentration of pro-inflammatory mediators, we do not distinguish between various types of inflammatory mediators such as MCP-1, inflammatory cytokines IL-1, endothelium stimulating cytokines and others. Instead, we group all pro-inflammatory mediators that induce positive chemotaxis and consider them as a single concentration. To include the effects of endothelium damage we defiine a function $f(t)$ (defined in equation (15)). The ox-LDL and ox-LDL enriched macrophages (foam cells) inside the intima also result in an increased concentration of pro-inflammatory mediators. Therefore, 
the concentration of inflammatory mediators is modelled by

$$
\frac{d c}{d t}=\beta f(t)+r_{L_{o x}} \frac{L_{o x}^{2}}{K_{L_{o x}}^{2}+L_{o x}^{2}}+r_{F} \frac{F^{2}}{K_{F}^{2}+F^{2}}-d_{c} c,
$$

where $\beta$ is the rate of production of inflammatory mediators from the initial endothelial damage and $d_{c}$ defines its natural decay rate. Here $r_{L_{o x}}$ and $r_{F}$ represent the production rates of inflammatory mediators induced by ox-LDL and foam cells, respectively. The parameters $\mathrm{K}_{\mathrm{L}_{\mathrm{ox}}}$ and $\mathrm{K}_{\mathrm{F}}$, respectively, represent the saturation constants for ox-LDL and foam cell. As discussed above, the injury to the endothelium is the primary cause of inflammatory mediators, which are manifestly increased by the subsequent pro-inflammatory process. Therefore, we expect two possible outcomes of inflammation: termination of the inflammatory process upon the replacement of the injured endothelial cell or the establishment of the self-perpetuating inflammatory process leading to the development of an atherosclerotic plaque.

Monocytes adhere to the site of the injury once triggered by the inflammatory mediators and penetrate the endothelial layer, reaching the arterial intima, where they differentiate into macrophages. T-lymphocytes and some other immune cells also penetrate the endothelial layer in response to the injury [5]. However, we ignore these immune cells in our model. Activated T-cells derived interferon gamma, with the assistance of macrophage colony stimulating factor, induces the conversion of monocytes into macrophages. We omit T-cells from our model by assuming that monocytes differentiate into macrophages immediately after they become trapped in the intima.

LDL migrate into the artery wall, alongside the immune cells, where they become oxidised (ox-LDL). Monocyte-derived macrophages generate activationdependent reactive oxygen species which are capable of in vivo oxidation of lipoproteins [14]. Endothelial cells also play a major role in the modification of LDL at the very early stages of lesion formation. However, macrophages that are trapped in the intima are the most prominent cell type to oxidise LDL within lesions, once atherosclerosis is initiated [15]. In our model, macrophages are the only cell type capable of the oxidative modification of LDL. 
Macrophages consume ox-LDL and transform into foam cells once they are heavily loaded, which eventually leads to the formation of an atheromatous plaque. Unlike other mathematical models $[12,11]$, we assume that the concentration of Ox-LDL never reaches the level where the ability of macrophages to ingest these particles is limited. Hence, we omit the saturating dynamics of the uptake of ox-LDL by the macrophages from our modelling. We also assume that the invaded LDL rapidly modify into ox-LDL, compared to timescales of ingestion of ox-LDL by the macrophages and other chemical reactions that are considered in this model, so we consider only modified LDL [12].

Activated macraphages, on being stimulated by ox-LDL, produce a large amount of anti-inflammatory cytokines, for example interleukin-10 (IL-10) [16]. These cytokines help abate lesion development by their various atheroprotective actions, such as inhibition of macrophage activation, production of pro-inflammatory mediators, and many other actions that play major roles in determining plaque stability [17]. HDL also inhibits plaque progression by various antiatherogenic actions. Its major roles include cholesterol efflux from foam cells, inhibition of the chemotaxis of monocytes, adhesion of leukocytes to the endothelium and endothelial dysfunction [18]. However, experimental studies suggest that these HDL particles fail to produce positive outcomes in controlling the development of atherosclerosis in the absence of IL-10 [19]. HDL particles, being activated by IL-10, can help reduce the plaque size by up to $60 \%$ in vivo $[19,17]$. To model the concentration of activated HDL, we consider that migrated HDL particles become activated by IL-10, which is released by macrophages when they are loaded with ox-LDL.

Using the above description of the mechanisms, we model the concentration 
of other species by

$$
\begin{aligned}
& \frac{d m}{d t}=\sigma_{m} c-\rho m-d_{m} m, \\
& \frac{d M}{d t}=\rho m-\mu M L_{o x}-d_{M} M, \\
& \frac{d L_{o x}}{d t}=\sigma_{L} M-\mu M L_{o x}-d_{L_{o x}} L_{o x}, \\
& \frac{d F}{d t}=\mu M L_{o x}-\mu_{1} F H-d_{F} F, \\
& \frac{d H}{d t}=\sigma_{H} F-\mu_{1} F H-d_{H} H .
\end{aligned}
$$

Equations (3)-(7) are dimensional model equations, and describe the follows processes. Due to the presence of inflammatory mediators, monocytes migrate into the intima at the rate $\sigma_{\mathfrak{m}}$, where they differentiate into macrophages at the rate $\rho$, and have a natural decay rate $d_{m}$. Macrophages consume ox-LDL at the rate $\mu$ to form foam cells, and have a natural decay rate $d_{M}$. LDL invade the intima at the rate $\sigma_{\mathrm{L}}$ and become modified in the presence of macrophages. Modified LDL is taken up by the macrophages at the rate $\mu$, and has a natural decay rate $d_{L_{o x}}$. HDL migrate into the intima at the rate $\sigma_{H}$ and become activated by IL-10, released upon the formation of foam cells. HDL removes foam cells at the rate $\mu_{1}$. The parameters $d_{F}$ and $d_{H}$ define the natural decay rates of foam cells and HDL, respectively. Table 1 lists all dimensional parameters in equations (2)-(7), along with their physical meanings.

The model (2)-(7) is nondimensionalised using the transformations: $\tilde{\mathrm{t}}=$ $\mathrm{d}_{\mathrm{L}_{o x}} \mathrm{t}, \mathrm{c}=\left(\mathrm{r}_{\mathrm{L}_{o x}} / \mathrm{d}_{\mathrm{L}_{o x}}\right) \tilde{\mathrm{c}}$ and $\mathrm{X}=\left(\sigma_{\mathrm{m}} \mathrm{r}_{\mathrm{L}_{\mathrm{ox}}} / \mathrm{d}_{\mathrm{L}_{o x}}^{2}\right) \tilde{X}$, where $\mathrm{X}=\left(\mathrm{m}, \mathrm{M}, \mathrm{L}_{\mathrm{ox}}, \mathrm{F}, \mathrm{H}\right)$ 
Table 1: Description of the parameters that appear in the biochemical model.

\begin{tabular}{|c|c|c|}
\hline Param & Description & Unit \\
\hline$\beta$ & $\begin{array}{l}\text { production rate of mediators from endothelial } \\
\text { damage }\end{array}$ & $\operatorname{pg} \mathrm{mm}^{-3} \mathrm{~s}^{-1}$ \\
\hline$r_{L_{o x}}$ & $\begin{array}{l}\text { production rate of mediators induced by ox- } \\
\text { LDL }\end{array}$ & 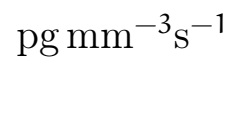 \\
\hline$r_{F}$ & $\begin{array}{l}\text { production rate of mediators induced by foam } \\
\text { cells }\end{array}$ & $\mathrm{pg} \mathrm{mm}^{-3} \mathrm{~s}^{-1}$ \\
\hline$\sigma_{\mathrm{m}}$ & migration rate of monocytes & $\mathrm{s}^{-1}$ \\
\hline$\sigma_{\mathrm{L}}$ & invasion rate of LDL & $\mathrm{s}^{-1}$ \\
\hline$\sigma_{\mathrm{H}}$ & migration rate of $\mathrm{HDL}$ & $s^{-1}$ \\
\hline$\rho$ & $\begin{array}{l}\text { rate at which monocytes differentiate into } \\
\text { macrophages }\end{array}$ & $\mathrm{s}^{-1}$ \\
\hline$\mu$ & rate at which macrophages consume ox-LDL & $\mathrm{mm}^{3} \mathrm{pg}^{-1} \mathrm{~s}^{-1}$ \\
\hline$\mu_{1}$ & rate at which HDL remove foam cells & $\mathrm{mm}^{3} \mathrm{pg}^{-1} \mathrm{~s}^{-1}$ \\
\hline $\mathrm{K}_{\mathrm{L}_{\mathrm{ox}}}$ & saturation constant for ox-LDL & $\mathrm{pg} \mathrm{mm} \mathrm{m}^{-3}$ \\
\hline$K_{F}$ & saturation constant for foam cells & $\operatorname{pg} \mathrm{mm}^{-3}$ \\
\hline$d_{c}$ & rate of decay of pro-inflammatory mediators & $\mathrm{s}^{-1}$ \\
\hline$d_{m}$ & rate of decay of pro-inflammatory mediators & $s^{-1}$ \\
\hline$d_{M}$ & rate of decay of macrophages & $s^{-1}$ \\
\hline$d_{L_{o x}}$ & rate of decay of ox-LDL & $\mathrm{s}^{-1}$ \\
\hline$d_{F}$ & rate of decay of foam cells & $\mathrm{s}^{-1}$ \\
\hline$d_{H}$ & rate of decay of HDL & $\mathrm{s}^{-1}$ \\
\hline
\end{tabular}


and $\tilde{X}=(\tilde{\mathrm{m}}, \tilde{\mathrm{M}}, \tilde{\mathrm{L}}, \tilde{\mathrm{F}}, \tilde{\mathrm{H}})$. Further, we define the dimensionless parameters

$$
\begin{aligned}
& \tilde{\beta}=\frac{\beta}{r_{L_{o x}}}, \quad \tilde{\alpha}=\frac{K_{L_{o x}} d_{L_{o x}}^{2}}{\sigma_{m} r_{L_{o x}}}, \quad \tilde{r_{F}}=\frac{r_{F}}{r_{L_{o x}}}, \quad \tilde{\sigma}=\frac{K_{F} d_{L_{o x}}^{2}}{\sigma_{m} r_{L_{o x}}}, \quad \tilde{\epsilon}=\frac{d_{c}}{d_{L_{o x}}}, \\
& \tilde{\rho}=\frac{\rho}{d_{L_{o x}}}, \quad \tilde{\kappa}=\frac{d_{m}}{d_{L_{o x}}}, \quad \tilde{\mu}=\frac{\mu \sigma_{m} r_{L_{o x}}}{d_{L_{o x}}^{3}}, \quad \tilde{\gamma}=\frac{d_{M}}{d_{L_{o x}}}, \quad \tilde{\delta}=\frac{\sigma_{L}}{d_{L_{o x}}}, \\
& \tilde{\mu}_{1}=\frac{\mu_{1} \sigma_{m} r_{L_{o x}}}{d_{L_{o x}}^{3}}, \quad \tilde{\phi}=\frac{d_{F}}{d_{L_{o x}}}, \quad \tilde{v}=\frac{\sigma_{H}}{d_{L_{o x}}}, \quad \tilde{\theta}=\frac{d_{H}}{d_{L_{o x}}} .
\end{aligned}
$$

When nondimensionalised, the six governing equations (2)-(7) become, after omitting the tildes,

$$
\begin{aligned}
& \frac{d c}{d t}=\beta f(t)+\frac{L^{2}}{\alpha^{2}+L^{2}}+r_{F} \frac{F^{2}}{\sigma^{2}+F^{2}}-\epsilon c, \\
& \frac{d m}{d t}=c-\rho m-\kappa m, \\
& \frac{d M}{d t}=\rho m-\mu M L-\gamma M, \\
& \frac{d L}{d t}=\delta M-\mu M L-L, \\
& \frac{d F}{d t}=\mu M L-\mu_{1} F H-\phi F, \\
& \frac{d H}{d t}=v F-\mu_{1} F H-\theta H,
\end{aligned}
$$

with transformed initial conditions $\mathrm{c}(0)=\mathrm{m}(0)=\mathrm{M}(0)=\mathrm{L}(0)=\mathrm{F}(0)=$ $H(0)=0$. In our model the function $f(t)$ includes the effects of periodic damage to the endothelium, and

$$
f(t)=\Theta(A \pi-t / Q) \sin ^{2}(t / Q),
$$

where $A$ defines the number of repeated cycles of injury to the endothelium, $Q=1 / d_{L_{o x}}$ and $\Theta(A \pi-t / Q)$ is the Heaviside function. Using the transformation $\tilde{\mathrm{t}}=\mathrm{d}_{\mathrm{L}_{\mathrm{ox}}} \mathrm{t}$, in nondimensional form

$$
f(t)=\Theta(A \pi-t) \sin ^{2}(t)
$$


Table 2: Dimensionless parameters that appear in the biochemical and plaque growth models. The parameters $\delta, \mu$ and $v$ are chosen for bifurcation analysis.

\begin{tabular}{ll|ll|ll} 
Param. & Value & Param. & Value & Param. & Value \\
\hline$\beta$ & $0.05[10]$ & $\alpha$ & 0.1 (estimated) & $\sigma$ & 0.1 (estimated) \\
$\epsilon$ & $1[10]$ & $\rho$ & $0.05[11]$ & $\gamma$ & $0.1[13]$ \\
$\mu_{1}$ & $0.25[13]$ & $\phi$ & $0.015[13]$ & $\theta$ & $0.05[13]$ \\
$r_{F}$ & $0.04[13]$ & $\kappa$ & $0.01[11]$ & & \\
$\delta$ & 0.2 & $\mu$ & 0.2 & $\nu$ & 0.3
\end{tabular}

where tildes are again omitted. One unit of dimensionless time corresponds to 12 hours in dimensional variables $\left(\mathrm{d}_{\mathrm{L}_{\mathrm{ox}}} \sim \frac{1}{12} \mathrm{~h}^{-1}\right)$ [11]. Therefore, one cycle $A=1$ of injury and recovery to the endothelium in the dimensionless system equates to approximately 36 hours worth of damage, which resembles the time course of recovery of endothelial function after reperfusion or mechanical injury [20]. Table 2 lists the dimensionless parameters, along with their values.

\subsection{Plaque Growth Model}

The plaque growth model is based on the concentration of the various species considered in the biochemical model that accumulate beneath the damaged part of the endothelial layer.

Define $V_{I}$ as the volume of the intima (bounded by the endothelial and internal elastic layers of the artery) before onset of atherosclerosis. This volume increases due to the accumulation of the various constituents of the plaque. Using the governing equations (9)-(13), the volume of the intima at time $t+\Delta t$ is approximated to first order in $\Delta t$ as by Bulelzai et al. [11],

$$
V(t+\Delta t)=V(t)+\sum_{X \in\left\{m, M, L_{o x}, F, H\right\}}\left(V_{x} \frac{d X}{d t}\right) V(t) \Delta t, \quad V(0)=V_{I},
$$

where $\mathrm{V}$ is the volume of the intima at time $t$, and $V_{m}, V_{M}, V_{L_{o x}}, V_{F}$ and $V_{H}$ 
are the volumes of each monocyte cell, macrophage cell, ox-LDL molecule, foam cell and HDL molecule, respectively. The inflammatory mediators are very small in size compared to other cells in the biochemical model, and are thus ignored. We recast equation (16) as a differential equation of the the intimal volume

$$
\frac{d V}{d t}=\sum_{X=m, M, L_{o x}, F, H}\left(V_{x} \frac{d X}{d t}\right) V, \quad V(0)=V_{I} .
$$

We then define $V=\tilde{V} V_{I}$ and introduce a dimensionless parameter $\eta=$ $\left(\sigma_{\mathrm{m}} \mathrm{r}_{\mathrm{L}_{\mathrm{ox}}} / \mathrm{d}_{\mathrm{L}_{\mathrm{ox}}}^{2}\right) \mathrm{V}_{\mathrm{F}}$ to recast equation (17) in the dimensionless form, where tildes are dropped,

$$
\frac{d V}{d t}=\frac{\eta V}{V_{F}} \sum_{X=m, M, L, F, H} V_{x} \frac{d X}{d t}, \quad V(0)=1
$$

To model the growth in the outward direction, we assume that the outer boundary of the plaque always takes the shape of a circular arc. Figure 1a and its caption define the various geometrical parameters.

It follows from the geometry that

$$
V=\frac{s^{2}}{\sin a}\left(\frac{a}{\sin a}-\cos a\right), \quad L=\frac{2 a s}{\sin a}, \quad h=\frac{s}{\sin a}(1-\cos a),
$$

where $\mathrm{V}$ is the volume of the circular segment bounded by the arc and the chord that represents the endothelial layer. The outward growth of the plaque is driven by the pressure $\mathrm{P}_{\mathrm{b}}$ exerted by the blood on the wall. However, the resistive pressure of the outer wall increases as the plaque continues to grow. The resistive pressure exerted by the outer wall is measured by

$$
\mathrm{P}=\frac{\mathrm{T}}{\mathrm{r}+\mathrm{h}} \approx \frac{\mathrm{T}}{\mathrm{r}}, \quad \mathrm{r} \gg \mathrm{h}
$$

where $T$ is the tension in the outer wall which is approximated by [24]

$$
\mathrm{T}=\lambda(\mathrm{L}-2 \mathrm{~s}),
$$


Figure 1: (a) An illustration of the plaque growth model. The arc length L, footprint radius $s$, contact angle $a$ and sagitta $h$ characterise the outward growth, and the basal drag $y(x, t)$ characterises the inward growth of the plaque, where $x$ represents the distance along the artery wall. Here $r$ designates the initial radius of the lumen. (b) Pressure load on endothelial layer $P_{\text {eff }}$ versus total volume of the plaque.

(a)

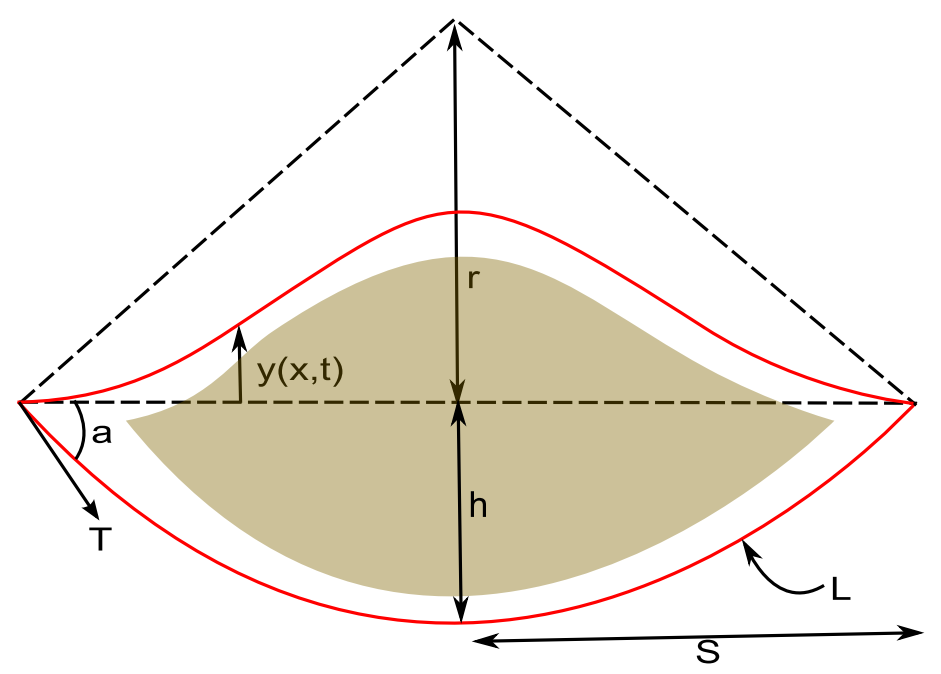

(b)

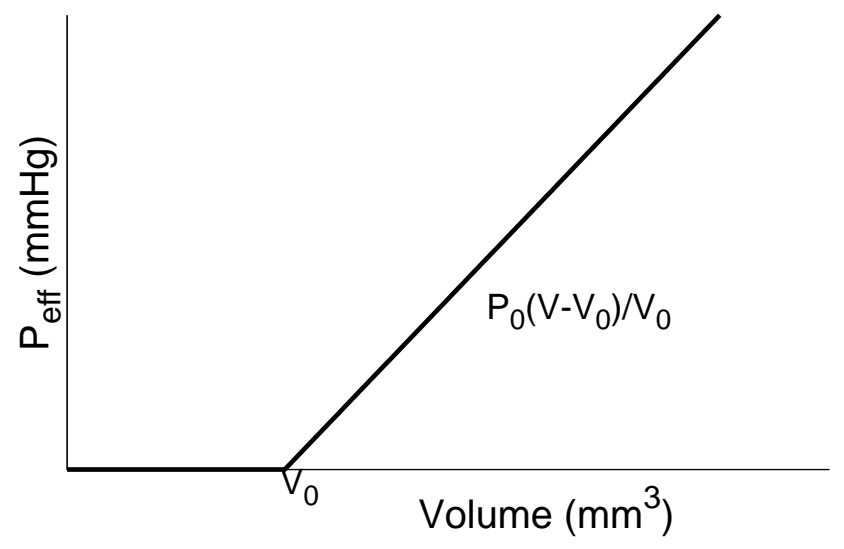


where $\lambda$ is Young's modulus of the outer wall. The outward growth stops when $P_{b}$ is surpassed by $P$. Hence, by substituting the condition $P=P_{b}$ along with equation (20) into equation (19), we obtain the threshold contact angle $a_{0}=\sqrt{3 P_{b} r / \lambda s}$, which determines the maximum plaque volume $V_{0}$ that the artery wall can compensate for by dilation.

Once the outward growth is stopped, further increase in plaque volume results in a pressure load $P_{\text {eff }}$ operating at the endothelial layer, as demonstrated in Figure $1 b$. The pressure load $P_{\text {eff }}$ shown in this figure defines the pressure potential per unit volume $V_{0}$ which is formulated using the linear end-diastolic pressure-volume relation. This pressure load induces inward growth of the plaque which is modelled by

$$
\frac{\partial y}{\partial x}=\frac{P_{\text {eff }}}{E}, \quad P_{\text {eff }}=\frac{P_{0} V_{\text {eff }}}{V_{0}},
$$

where, $\mathrm{P}_{0}=\frac{\mathrm{T}_{0}}{\mathrm{r}}=\mathrm{P}_{\mathrm{b}}$ and

$$
V_{\text {eff }}= \begin{cases}V-V_{0} & \text { if } V>V_{0} \\ 0 & \text { if } V<V_{0}\end{cases}
$$

Equation (21) is supplemented by the boundary conditions $y( \pm s)=\frac{\partial y}{\partial x}( \pm s)=$ 0 . The parameters appearing in the plaque growth model are listed in Table 3.

\section{Results and discussion}

\subsection{Simulations of the dynamics}

We first solve the biochemical model using the ode15s routine in Matlab. Table 2 summarises the parameter values used in the numerical simulations, unless stated otherwise. Figure 2 presents the dimensionless concentrations of the pro-inflammatory mediators $\mathrm{c}$, and the species $\mathrm{m}, \mathrm{M}, \mathrm{L}, \mathrm{F}$ and $\mathrm{H}$. This 
Table 3: List of parameters that appear in the plaque growth model.

\begin{tabular}{lll} 
Param. & Description & Value \\
\hline$V_{\mathrm{m}}$ & Mean cell volume of monocytes & $10^{-17} \mathrm{~mm}^{3}[11]$ \\
$V_{M}$ & Mean cell volume of macrophages & $10^{-14} \mathrm{~mm}^{3}[11]$ \\
$V_{L_{\mathrm{ox}}}$ & Mean volume of ox-LDL particles & $10^{-21} \mathrm{~mm}^{3}[11]$ \\
$V_{\mathrm{F}}$ & Mean cell volume of foam cells & $10^{-08} \mathrm{~mm}^{3}[11]$ \\
$\mathrm{V}_{\mathrm{H}}$ & Mean volume of HDL particles & $10^{-16} \mathrm{~mm}^{3}[21]$ \\
$\lambda$ & Young's modulus of the adventi- & $900 \mathrm{kPa}[22]$ \\
& tial layer & \\
$\mathrm{E}$ & Young's modulus of the endothe- & $24.5 \mathrm{kPa}[23]$ \\
& lial layer & $120 \mathrm{mmHg}$ \\
$\mathrm{P}_{\mathrm{b}}$ & Normal blood pressure & $0.6[12,13]$ \& estimated \\
$\eta$ & Dimensionless parameter
\end{tabular}

figure illustrates that, following a single cycle of injury to the endothelium $(A=1)$, we get a healthy outcome; the release of inflammatory mediators stops as cells are removed and the injured endothelial cell is replaced by a healthy one.

However, an inflammatory response is observed for repeated injuries (say $A=4$, Figure 3). The increased initial concentration of inflammatory mediators from repeated injuries results in an increased level of other cells, which ultimately results in increasing the concentrations of inflammatory mediators and thereby establishes a cycle of inflammation. Consequently, the system settles to a non-zero equilibrium which physiologically relates to chronic inflammation as the inflammatory process remains active. The system also behaves in a qualitatively similar manner to $A=1$ and a sufficiently large value of $\delta$ (not shown). This is equivalent to high blood LDL cholesterol levels and hence agrees strongly with the pathophysiology [7]. 
Figure 2: Numerical solutions for $\delta=0.2>\delta_{\mathrm{c}}$ and $A=1$. The inflammatory process induced by a single cycle of injury $(A=1)$ to the endothelium is not strong enough to drive the development of an atherosclerotic lesion: the release of inflammatory mediators stops upon the removal of all cells and hence, the damage is successful repaired.
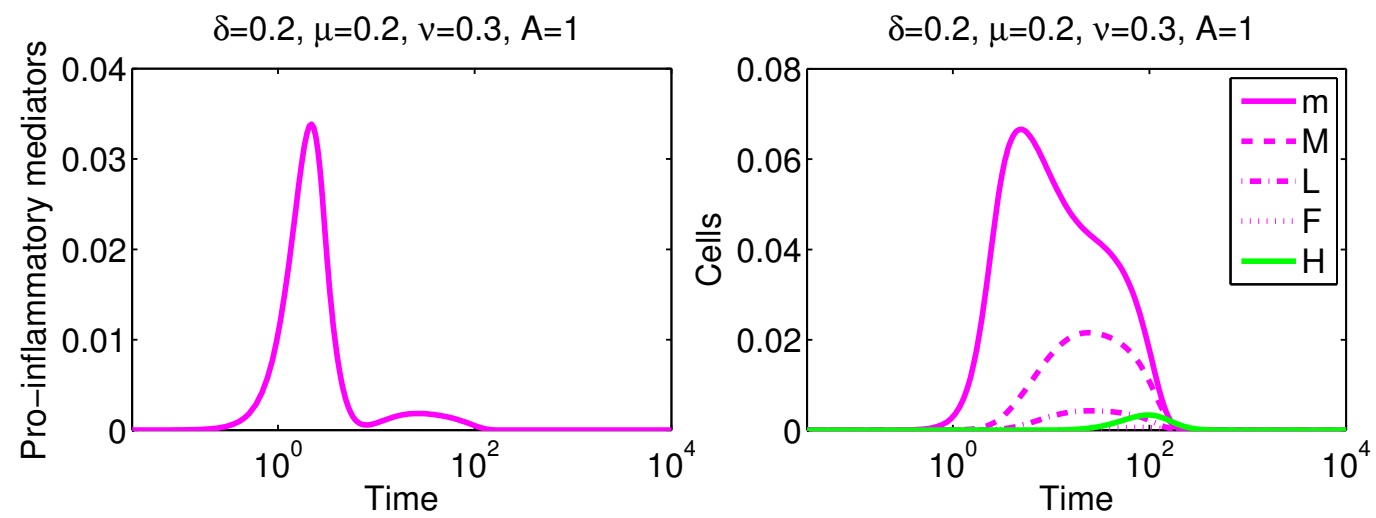

Figure 3: Numerical solutions for $\delta=0.2>\delta_{c}$ and $A=4$. An increased initial concentration of inflammatory mediators from repeated injuries $(A=4)$ results in an increased level of other cells, which ultimately results in increasing the concentrations of inflammatory mediators and thereby establishes a cycle of inflammation.
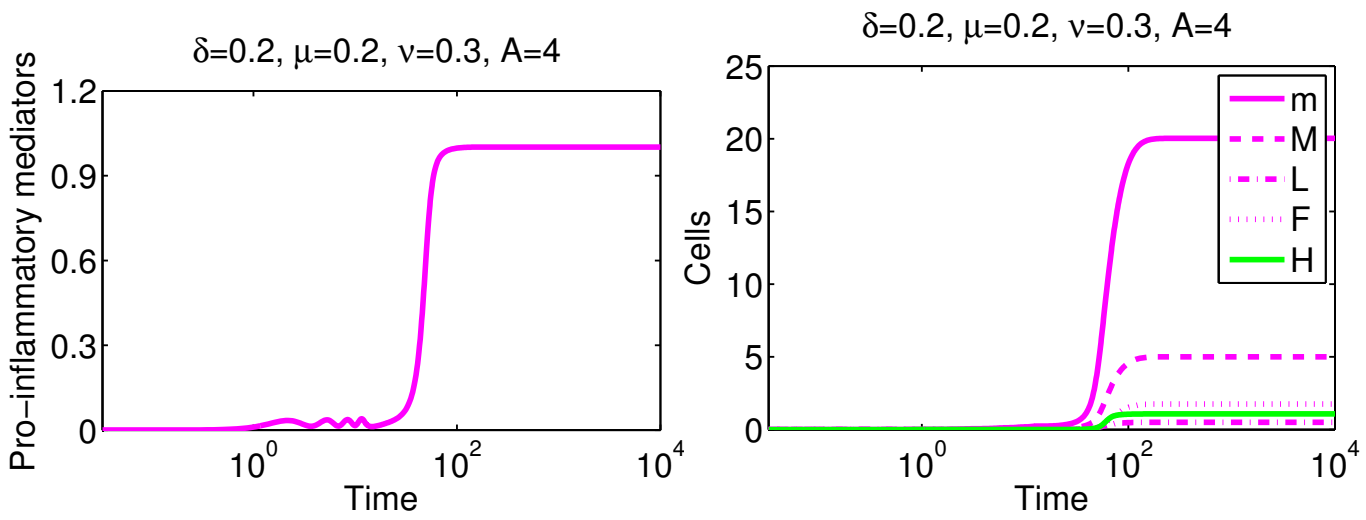
Figure 4: Numerical solutions for a large uptake rate $\mu$ of ox-LDL by macrophages. The pro-inflammatory process induced by ox-LDL subsides greatly for a large $\mu$ but a relatively weak inflammatory process induced by foam cells remains active.
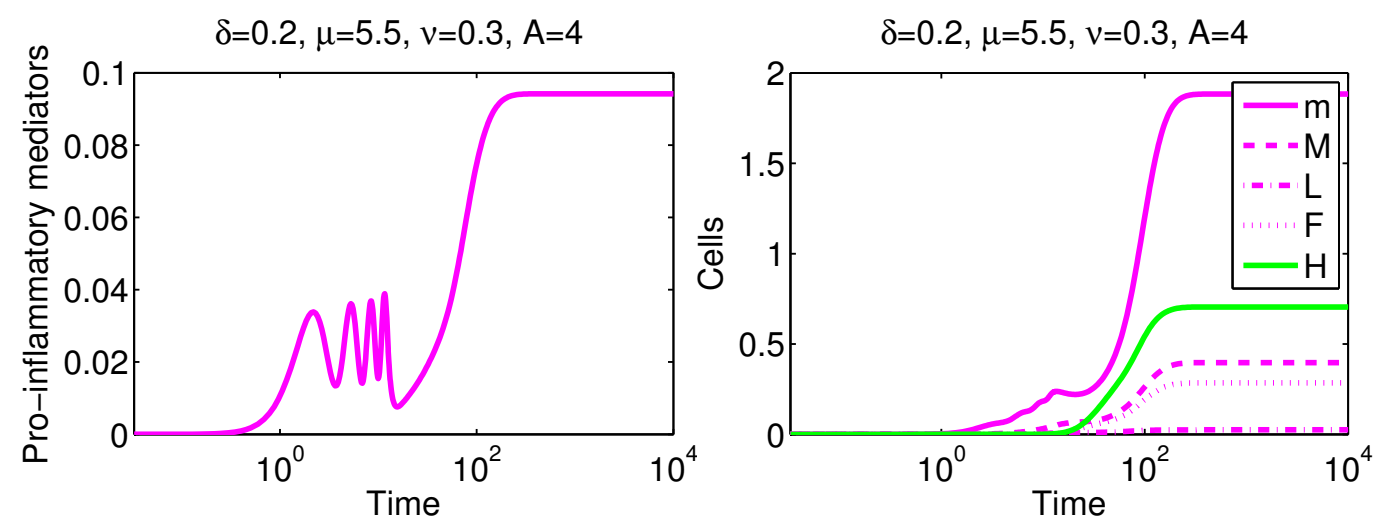

Figure 5: For sufficiently large values of $\mu$ and $\nu$, ox-LDL and foam cells are successfully removed from the lesion, hence the system reverts to the healthy state.
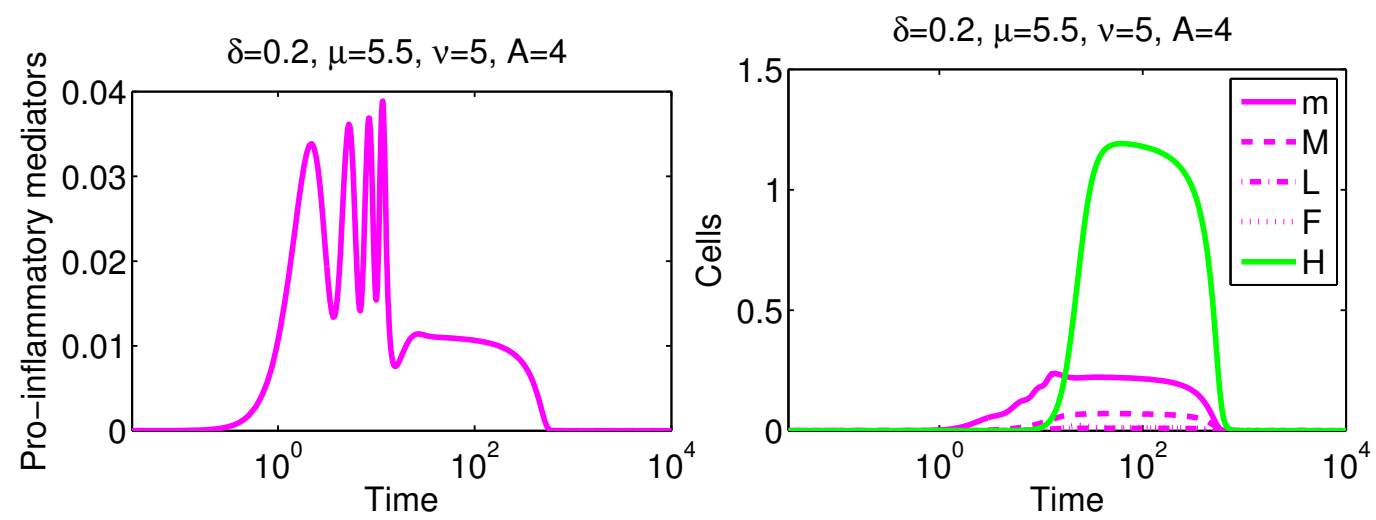


\subsection{Bifurcation analysis}

The bistability criteria of the system, as discussed in Section 3.1, is confirmed by the bifurcation analysis. The system of equations (8)-(13) always has a trivial steady state which is stable for all parameter values. However, when the value of $\delta$ is increased beyond a threshold $\delta_{c} \sim 0.034$ (calculated in MatCont [25]), the system possesses another eleven steady states (calculated using Maple). However, only two of these states are biologically reasonable; one is stable and the other one is a saddle point. The solid branch of the equilibrium concentration of inflammatory mediators curve, shown in Figure 6a, is stable, while the dashed branch is unstable.

To gain a better understanding of the behaviour of the system, we study the bifurcations with increased biological complexity. We first assume that there are no foam cells and no HDL inside the intima, that is, $F=0$ so $r_{\mathrm{F}}=0$, and $\mathrm{H}=0$. The system reduces to a $4 \times 4$ system which is bistable if $\delta>\frac{\mu \epsilon(\rho+\kappa)}{\rho} \alpha^{2}$. The parameter space for $\delta$ and $\mu$, presented in Figure 6b, shows that the system is monostable for a sufficiently large value of $\mu$ (uptake rate of ox-LDL by the macrophages), which implies that the system returns to a healthy state when ox-LDL are removed from the lesion.

We now assume that there are foam cells but no HDL inside the intima, that is $\mathrm{F} \neq 0$ so $\mathrm{r}_{\mathrm{F}} \neq 0$, and $\mathrm{H}=0$. In this case, the bistability of the system no longer depends on the value of $\mu$ (Figure 6c). For large $\mu$ the pro-inflammatory process induced by ox-LDL subsides greatly but still a relatively weak inflammatory process induced by foam cells remains active. Low level of cells and inflammatory mediators are observed following this weak inflammation (Figure 4).

Finally, assume that $F \neq 0$, so $r_{F} \neq 0$, and $H \neq 0$. The bifurcation diagram presented in Figure 6d demonstrates that an increasing value of $v$ brings the limit point curve (the curve dividing the parameter space) back to its original position. Therefore, corresponding to sufficiently large values of $\mu$ and $\nu$, ox-LDL and foam cells are successfully removed from the lesion, hence we get 
Figure 6: Bifurcation plots showing the (dimensionless) equilibrium proinflammatory mediators concentration as a function of the invasion rate of LDL $\delta$ in (a), and the parameter spaces corresponding to different biological scenarios for $\delta$ and $\mu$ in (b) $-(\mathrm{d})$.

(a) Limit point.

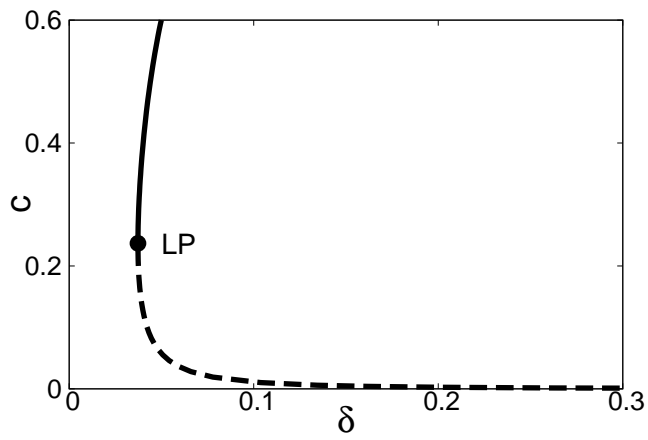

(c) $\mathrm{F} \neq 0, \mathrm{r}_{\mathrm{F}} \neq 0, \mathrm{H}=0$.

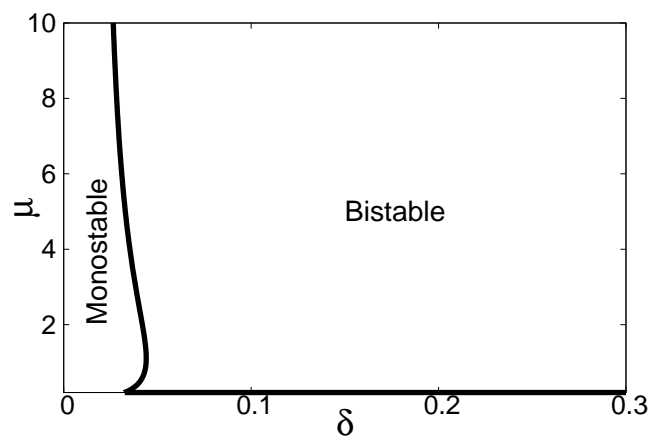

(b) $\mathrm{F}=0, \mathrm{r}_{\mathrm{F}}=0, \mathrm{H}=0$.

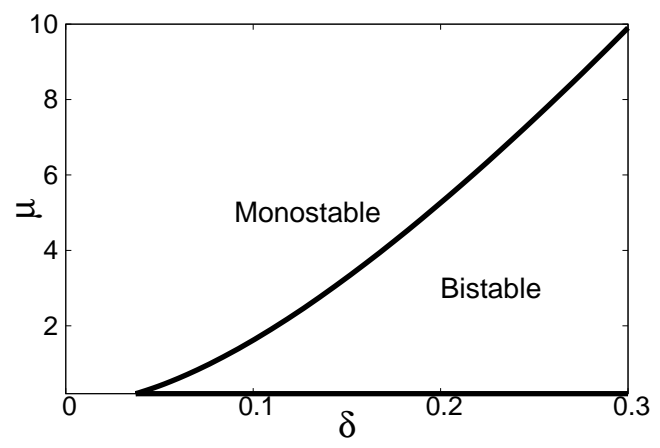

(d) $\mathrm{F} \neq 0, \mathrm{r}_{\mathrm{F}} \neq 0, \mathrm{H} \neq 0$.

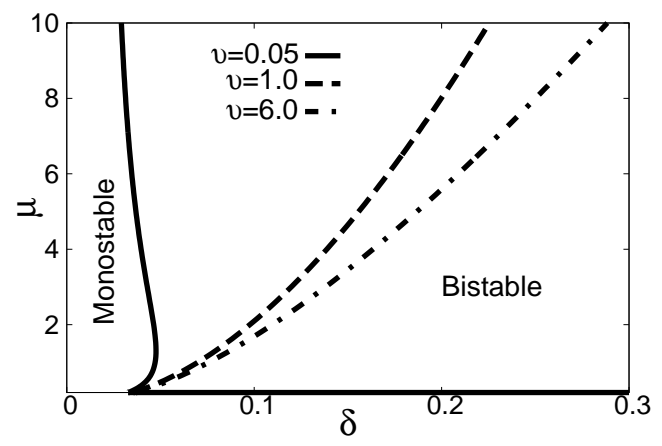

a healthy outcome, as shown in Figure 5. 
Figure 7: Plaque shapes at different dimensionless times.

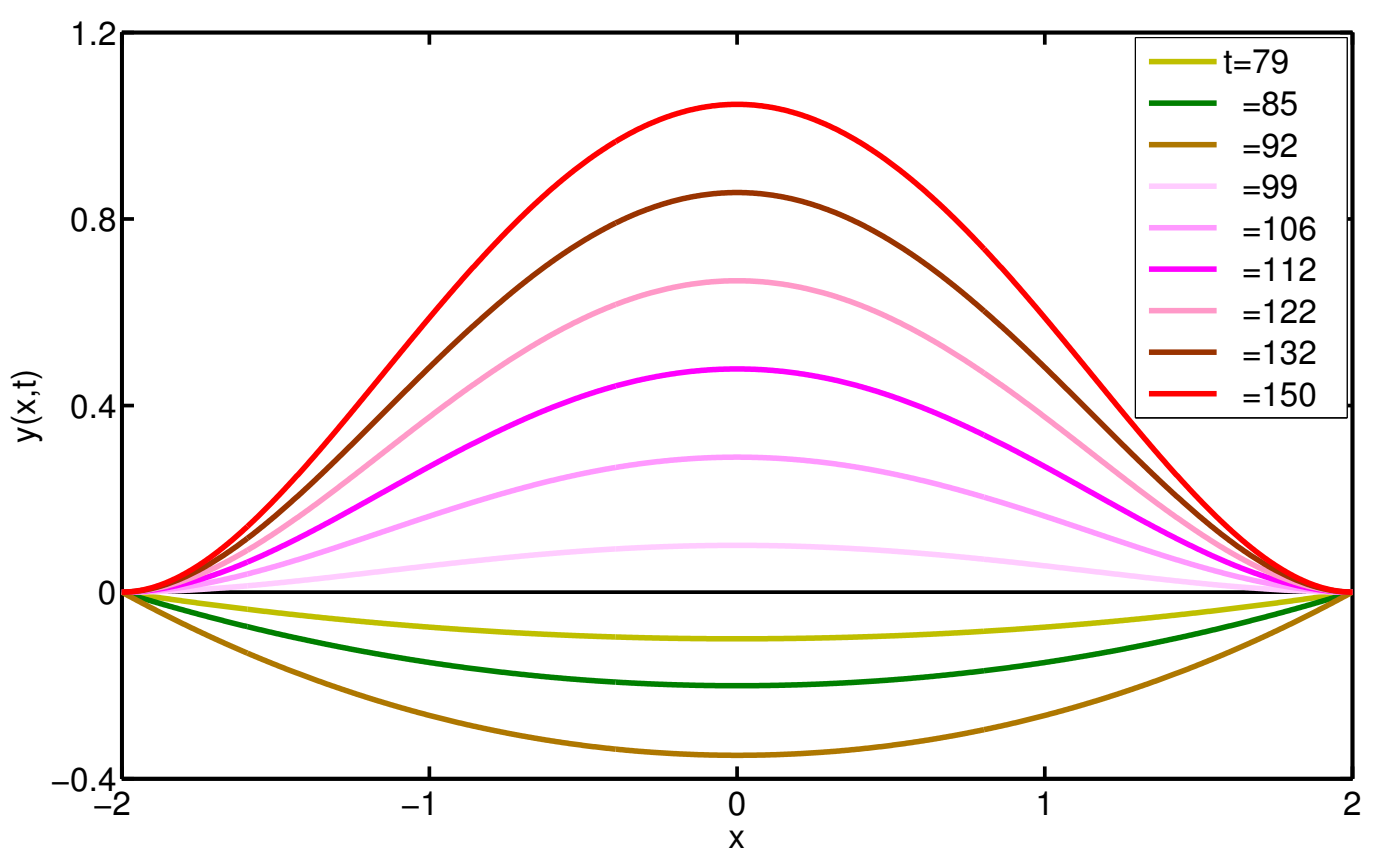

\subsection{Plaque growth simulations}

We solve the plaque growth model using the values corresponding to the outcome of the biochemical model that are shown in the Figure 3. The lumen radius $r$ and the footprint radius $s$ are both considered to be $2 \mathrm{~mm}$. The plaque volume $\mathrm{V}$ at different times, obtained by integrating equation (18), is used to simulate the plaque growth. The plaque growth towards the outer wall occurs as long as $\mathrm{V}<\mathrm{V}_{0}$, where the boundary always takes the shape of a circular arc, as shown by the curves below the zero axis in Figure 7 . However, an artery in a patient with low physical activity, high cholesterol and high blood pressure may fail to produce this outward growth by dilation due to the artery's high stiffness.

The curves above the zero axis in Figure 7 show the shape of the plaque cap 
at different positions and times. The plaque starts to grow into the lumen when $\mathrm{V}>\mathrm{V}_{0}$; however, this growth stops when the cells considered in the biochemical model settle to their equilibrium values.

\section{Conclusions}

We presented a mathematical model of the formation of an atherosclerotic plaque based on the response-to-injury hypothesis of atherosclerosis, which was originally proposed by Ross [26]. The compensatory response of the immune system initiates a series of complex biochemical and cellular processes in response to an injury, which may result in the development of an atherosclerotic lesion if the immune system becomes corrupted and fails to replace the injured endothelium successfully. This failure occurs because of an imbalance between the pro- and anti-inflammatory actions taken by some cells and cellular species that are relevant to the repair mechanism and lesion development. Elevated levels of modified low density lipoprotein (ox-LDL) in the vessel wall is one of the major factors that instigates this failure by triggering a self-accelerating inflammatory process.

Section 2.1 developed a mathematical model describing the earliest events in the formation of an atherosclerotic plaque. Analysis of the model shows that, following an injury to the endothelium, an atherosclerotic plaque is initiated if the migration rate of LDL cholesterol exceeds a threshold value, which strongly complies with the pathophysiology of atheroslcerotic plaques initiation $[7,6]$. A similar threshold value for LDL migration was also suggested by Bulelzai and Dubbeldam [11], and Chalmers et al. [12]. These studies [11, 12] also suggested a minimum migration rate of LDL for a self-accelerating inflammatory process to be initiated. However, the threshold values suggested in these studies are numerically higher than those found from our model, apparently due to the exclusion of the effects of endothelial injury in their modelling. We found that a comparatively low migration rate of LDL can drive the development of atherosclerosis if the endothelium undergoes repetitive injuries. 
Formation of foam cells is considered to be a major hallmark of early stage atherosclerotic lesions [16]. The series of bifurcation analyses conducted in Section 3.2 demonstrates that a large uptake rate of ox-LDL by macrophages results in a reduced abundance of ox-LDL particles in the lesion. However, the self-accelerating inflammatory process still remains active due to the proinflammatory cytokines that are produced during the formation of foam cells. Our study also includes activated high density lipoprotein (HDL) particles, assumed to be activated in the presence of anti-inflammatory cytokines (IL-10) and produced during the formation of foam cells. We found that raising the migration rate of HDL reduces the severity of the inflammation, and this inflammation may completely stop for a sufficiently large migration rate of HDL.

Atherosclerosis arises as the consequence of multiple dynamical cell processes. Our model provides improved qualitative and quantitative insights into the resolution of inflammation. Analysis of this model suggests that a single therapeutic target, such as lowering the blood cholesterol level (LDL) or raising the good cholesterol level (HDL) may not produce the expected outcome of preventing atherosclerosis. Multiple therapeutic targets, including improvement of endothelium health, may be required to produce the desired outcome. However, our model does not include the formation of a necrotic core or fibrous cap. To make the model more quantitatively reliable, several other cell types, such as smooth muscles cells, T-cells and some other cellular species that take part in the development of complex atherosclerotic lesions and induce the plaque cap disruption, need to be taken into consideration.

Another novel aspect of our study is the inclusion of both inward and outward expansions of the arterial wall in plaque growth modelling. This plaque growth model can be coupled with a more sophisticated mathematical model of later-stage atherosclerotic plaque to investigate mechanical properties of the plaque. 


\section{References}

[1] M. J. van Gils and D. Vukadinovic, A. C. van Dijk, D. W. J. Dippel, W. J. Niessen and A. van der Lugt. Carotid atherosclerotic plaque progression and change in plaque composition over time: A 5-year follow-up study using serial CT angiography. Am. J. Neuroradiol., 37(7):1267-1273, 2012. doi:10.3174/ajnr.A2970 C321

[2] M. Naghavi, P. Libby, E. Falk, et al. From vulnerable plaque to vulnerable patient; A call for new definitions and risk assessment strategies: Part I. Circulation, 108(14):1664-1672, 2003. doi:10.1161/01.CIR.0000087480.94275.97 C322

[3] T. F. Lücher and M. Barton. Biology of the Endothelium. Clin Cardiol., 20(Supp. 2):3-10, 1997. https://www.researchgate.net/profile/ Matthias_Barton/publication/13807118_Biology_of_the_ endothelium/links/0046352c54fe700895000000.pdf C322, C324

[4] E. Mannarino and M. Pirro. Endothelial injury and repair: A novel theory for atherosclerosis. Angiology, 59:69S-72S, 2008. doi:10.1177/0003319708320761 C322, C324

[5] C. Davis, J. Fischer, K. Ley and I. J. Sarembock. The role of inflammation in vascular injury and repair. J. Thromb. Haemost., 1(8):1699-1709, 2003. doi:10.1046/j.1538-7836.2003.00292.x C322, C325

[6] G. K. Hansson and P. Libby. The immune response in atherosclerosis: a double-edged sword. Nat. Rev. Immunol., 6(7):508-519, 2006. doi:10.1038/nri1882 C322, C340

[7] R. Ross. Atherosclerosis - an inflammatory disease, New Engl. J. Med., 340(2):115-126, 1999. doi:10.1056/NEJM199901143400207 C322, C334, C340

[8] B. F. Asztalos. High-density lipoprotein metabolism and progression of atherosclerosis: new insights from the HDL atherosclerosis treatment 
study. Curr. Opin. Cardiol., 19(4):385-391, 2004.

http://journals. lww.com/co-cardiology/Abstract/2004/07000/ High_density_lipoprotein_metabolism_and.16.aspx C322

[9] N. El Khatib, S. Genieys and V. Volpert. Atherosclerosis initiation modeled as an inflammatory process. Math. Model. Nat. Phenom., 2(2):126-141, 2007. doi:10.1051/mmnp:2008022 C323

[10] P.-W. Fok. Mathematical model of intimal thickening in atherosclerosis: Vessel stenosis as a free boundary problem. J. Theor. Biol., 314:23-33, 2012. doi:10.1016/j.jtbi.2012.07.029 C323, C330

[11] M. A. K. Bulelzai and J. L. A. Dubbeldam. Long time evolution of atherosclerotic plaques, J. Theor. Biol., 297:1-10, 2012. doi:10.1016/j.jtbi.2011.11.023 C323, C326, C330, C334, C340

[12] A. D. Chalmers, A. Cohen, C. A. Bursill and M. R. Myerscough. Bifurcation and dynamics in a mathematical model of early atherosclerosis. J. Math. Biol., 71:1451-1480, 2015. doi:10.1007/s00285-015-0864-5 C323, C326, C334, C340

[13] A. Friedman and W. Hao. A mathematical model of atherosclerosis with reverse cholesterol transport and associated risk factors. B. Math. Biol., 77(5):758-781, 2015. doi:10.1007/s11538-014-0010-3 C323, C330, C334

[14] G. M. Chisolm, S. L. Hazen, P. L. Fox and M. K. Cathcart. The oxidation of lipoproteins by monocytes-macrophages: Biochemical and biological mechanisms. J. Biol. Chem., 274(37):25959-25962, 1999. doi:10.1074/jbc.274.37.2595 C325

[15] A. Daugherty, N. R. Webb, D. L. Rateri and V. L. King. Thematic review series: The immune system and atherogenesis. Cytokine regulation of macrophage functions in atherogenesis, J. Lipid Res., 46(9):1812-1822, 2005. doi:10.1194/jlr.R500009-JLR200 C325

[16] L. J. H. van Tits, R. Stienstra, P. L. van Lent, M. G. Netea, L. A. B. Joosten and A. F. H. Stalenhoef. Oxidized LDL enhances 
pro-inflammatory responses of alternatively activated M2 macrophages: A crucial role for Kruppel-like factor 2. Atherosclerosis, 214(8):345-349, 2011. doi:10.1016/j.atherosclerosis.2010.11.018 C326, C341

[17] Z. Mallat, S. Besnard, M. Duriez, et al. Protective role of interleukin-10 in atherosclerosis. Circ. Res., 85(8):e17-e24, 1999. doi:10.1161/01.RES.85.8.e17 C326

[18] P. J. Barter, S. Nicholls, K.-A. Rye, G. M. Anantharamaiah, M. Navab and A. M. Fogelman. Antiinflammatory properties of HDL, Circ. Res., 95(8):764-772, 2004. doi:10.1161/01.RES.0000146094.59640.13 C326

[19] M. Sanson, E. Distel and E. A. Fisher. HDL induces the expression of the M2 macrophage markers arginase 1 and Fizz-1 in a STAT6-dependent process. PLoS One, 8(8):e74676, 2013. doi:10.1371/journal.pone.0074676 C326

[20] L. Tilling, J. Hunt, A. Donald, B. Clapp and P. Chowienczyk. Arterial injury and endothelial repair: Rapid recovery of function after mechanical injury in healthy volunteers. Card. Res. Prac., 2014:367537, 2014. doi:10.1155/2014/367537 C330

[21] F. Alexandrea, V. H. S. Zagoa, N. B. Panzoldo, et al. Reference values for high-density lipoprotein particle size and volume by dynamic light scattering in a Brazilian population sample and their relationships with metabolic parameters. Clin. Chim. Acta, 442:63-72, 2015. doi:10.1016/j.cca.2015.01.006 C334

[22] T. Khamdaeng, J. Luo, J. Vappou, P. Terdtoon, E. E. Konofagou. Arterial stiffness identification of the human carotid artery using the stress-strain relationship in vivo. Ultrasonics, 52(3):402-411, 2012. doi:10.1016/j.ultras.2011.09.006 C334

[23] T. G. Kuznetsova, M. N. Starodubtseva, N. I. Yegorenkov, S. A. Chizhik, R. I. Zhdanov. Atomic force microscopy probing of cell elasticity, Micron., 38(8):824-833, 2007, doi:10.1016/j.micron.2007.06.011 C334 
[24] J. Bell, C. Breward, T. Chou, P.-W. Fok, J. M. Haugh, Q. Li, L. Rossi, A. Walter, X. Yang, A. Zemlyanova and N. Zhang. Mathematical models for vulnerable plaques. Technical Report, 2009. http://www. maths-in-industry.org/miis/272/ C331

[25] A. Dhooge, W. Govaerts and Y. A. Kuznetsov. MATCONT: a MATLAB package for numerical bifurcation analysis of ODEs. ACM T. Math. Softw. (TOMS), 29(2):141-164, 2003. doi:10.1145/779359.779362. C337

[26] R. Ross. The pathogenesis of atherosclerosis: A perspective for the 1990s. Nature, 362:801-809, 1993. doi:10.1038/362801a0. C340

\section{Author addresses}

1. Md Hamidul Islam, School of Natural Sciences, Griffith University, Nathan, Queensland, Australia.

mailto:mdhamidul.islam@griffithuni.edu.au

2. P. R. Johnston, School of Natural Sciences, Griffith University, Nathan, Queensland, Australia.

mailto:p.johnston@griffith.edu.au 